\title{
Using Tangible Drawing Tools on a Capacitive Multi-touch Display
}

\author{
Rachel Blagojevic, Xiliang Chen, Ryan Tan, Robert Sheehan, Beryl Plimmer \\ Department of Computer Science \\ University of Auckland \\ Private Bag 92019, Auckland 1142, New Zealand \\ \{rpat088|xche185|rtan052\}@aucklanduni.ac.nz, \{r.sheehan@|beryl@cs.\}auckland.ac.nz
}

\begin{abstract}
We present an innovative drawing tool that can detect tangible drawing instruments on a capacitive multi-touch tablet. There are three core components to the system: the tangible hardware, the recognizer used to identify the tangibles, and the drawing application. Our tangible drawing instruments include a ruler, protractor and set square. Users can apply these familiar physical instruments to construct digital ink drawings on a tablet in an intuitive and engaging manner. The user evaluation shows that the tangible drawing tools are easy to use and have a high rate of recognition on the touch screen.
\end{abstract}

TUI, tangible, multi-touch, physical interaction, interactive surface, drawing tools.

\section{INTRODUCTION}

We have developed an innovative drawing application for use on Apple iPads and Windows tablets with the ability to detect tangible drawing instruments. To do this we have constructed tangibles, developed a recognizer to detect tangibles on a capacitive touch display, and built a drawing application bringing these components together.

An already popular tangible drawing instrument for digital content is the stylus, which mimics a real pen. We have added to this by creating other recognizable drawing instruments, including a ruler, protractor and set square. Users can apply these familiar physical instruments when constructing digital ink drawings on a multi-touch tablet. The tangibles provide a novel and intuitive way to construct and interact with digital drawings. To our knowledge, tangible drawing tools (other than styli) have never been used on capacitive touch displays for manipulating electronic content. This work is an exploration into how such a system could work.

A skill acquired early in life is the ability to manipulate tangible objects. This skill has long been identified in mathematics education (Marzola 1987; Uttal et al. 1997). Using tangibles for computing operations takes advantage of this existing skillset: pen-based interaction is a testament to this. The tactile feedback offered by tangibles allows users to take advantage of their natural sense of touch while enjoying the benefits of computer based applications.
The use of tangibles on touch displays have been shown to improve interaction with interface objects as they are easier to manipulate, acquire and control in comparison to virtual objects (Tuddenham et al. 2010). The use of tangibles has also been shown to improve collaborative work (Stanton et al. 2001; Olson et al. 2010). We seek to enhance user experience with drawing applications by exploiting the benefits of tangible interaction.

Small factor touch sensitive devices such as phones and tablets typically have capacitive displays, larger touch devices, such as tabletop displays, have camera based detectors. Tangibles on tabletops have been explored for many years (Wellner 1991; Fitzmaurice 1995; Ullmer et al. 1997). Tabletops typically use cameras to capture the position of the tangible; this requires specialist equipment and environments. The advantage of capacitive touch is that it is readily available in portable tablets requiring no special environment. Using capacitive touch alters the recognition approach from image processing to gesture processing of the touch points. Current tablets (e.g. Apple iPads) can synchronously register 10 or more touch-points, these points may be fingertips or any conductive material (Yu et al. 2011). As an extension of recent techniques (Yu et al. 2011) we have devised a novel approach which enables tangible drawing tools to work with capacitive displays.

The system we have developed is highly innovative - very little work has been done in this area previously. Our goal is to assess whether it has 
further potential by evaluating its usability. In particular, we want to determine whether the tangible drawing tools can be used to construct simple drawings in an intuitive manner. Our future goal is to add further intelligence requiring unique identification of each tool. With these enhancements we believe this system has the potential to provide far more benefits than using standard drawing tools on a screen or paper.

\section{RELATED WORK}

Physical objects are beneficial for learning (Inhelder et al. 1964). For example, manipulatives have been used for years to assist in mathematics education (Marzola 1987; Uttal et al. 1997). Similarly tangibles are a particularly useful tool for children when interacting with technology because of the added physical dimension (Fails et al. 2005).

The use of tangibles on touch surfaces have been shown to improve interaction with interface objects. Tuddenham et al (2010) compared the use of tangibles and virtual objects on multi-touch interfaces. They found that when using the tangible objects significantly fewer errors were made and significantly less time was taken to acquire and manipulate the objects.

Few studies have been done in the area of tangibles on capacitive touch screens. Yu et al (2011) present three tangible technologies for use on capacitive touch surfaces: the first employs four point patterns for object identification, the second use a modulation circuit to generate touches of varying frequency, and the third is a hybrid of the previous designs. Our observation is that these tangibles are large in size - particularly thickness, in comparison to our tangibles. They report that the size of the circuit board alone is $2 \times 3 \times 3 \mathrm{~cm}$.

AppMATes $^{1}$ are another example of tangible objects, in the form of toy cars that can be used on the Apple iPad. The cars are uniquely identifiable using touch point patterns. More recently (Chan et al. 2012) stackable tangibles, sliders and dials for capacitive screens were introduced. The stackable tangibles are able to sense changes in capacitance when blocks are placed on top of them - this in turn modifies the touch point pattern for identification. The dials and sliders are made of conductive zebra rubber and also use unique touch point patterns.

Although the use of tangibles and multi-touch has been investigated tangible drawing tools with multitouch capacitive screens are yet to be explored. This combination presents significant challenges in the hardware, recognizers, and the interaction design of the applications to ensure that they provide a good user experience.

\footnotetext{
${ }^{1}$ http://www.appmatestoys.com/
}

\section{TANGIBLE HARDWARE}

Our goal is to create recognizable drawing instruments on a capacitive touch display that are easy to interact with. Capacitive screens are designed specifically to detect the electrical properties of fingertips. Hence we use conductive materials that can carry an electrical pulse from the user's fingertips to the touch screen.

There were three iterations in our design process for constructing the tangible hardware. For the first iteration our goal was to produce an instrument that could be detected by the touch screen. We built a recognizable ruler using aluminum foil (conductive material) and tape (Figure 1). Three balls made of foil served as the touch points (Figure 1b). The touch points were 'wired' together using more aluminum foil (Figure 1a) - the users finger has to be in contact with the 'wire'. Although recognition of this tangible was possible on the touch screen, a significant amount of pressure had to be applied before the touch points were detected. This indicated that the screen to tangible, and tangible to body contact needed improvement.

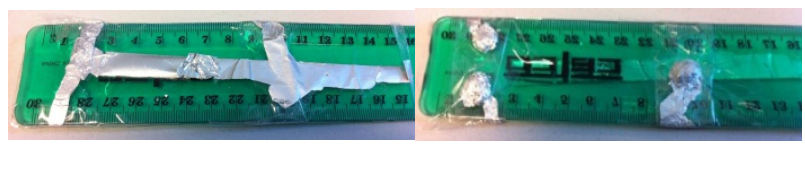

a. Top b. Bottom

Figure 1: First iteration tangible design

For the second iteration our goal was to improve the points of contact that were hindering user interaction with the device and to discriminate between multiple tangibles. Three tangibles were made: a ruler, protractor, and set square (Figure 2). To discriminate between the tangibles each required a unique ID. The touch points were placed in unique triangular patterns on the bottom of the tangibles. The distance between each pair of touch points served as the tangible ID. This ID allowed us to differentiate between multiple tangible objects, and calculate their orientation and location (see the next section for a full explanation).

To improve the screen to tangible contact, touch points were made of the conductive rubber (cut from styli tips) fitted onto steel nuts and bolts (Figure $2 \mathrm{~b}$ ). The points were connected together with copper wire (Figure 2a). This provided better conductivity of the points and therefore improved the detection of the tangible. The rubber also

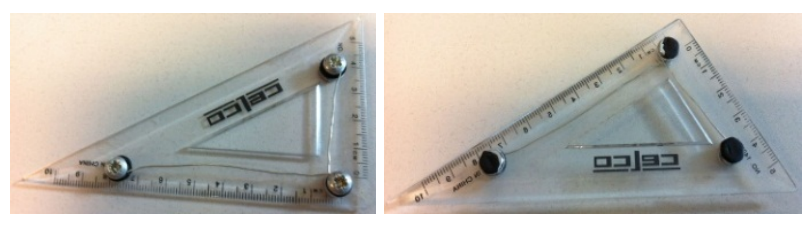
a. Top
b. Bottom

Figure 2: Second iteration tangible design 
added friction between the tangible and the screen, preventing unwanted movements when drawing.

After preliminary testing we found that the tangibles needed better stability for more consistent detection. The gap between the screen and tangible should be minimized by keeping the points as flat as possible and positioned such that when the tangible is moved all points stay in contact with the screen. Instability was a problem particularly for the protractor due to touch point locations. In terms of the tangible to body contact, using the screws improved this, giving users a clear point of contact.

In the final iteration, tangibles were constructed based on the above findings; these included a ruler, set square, and protractor (Figure 3 ). For the screen to tangible contacts the whole tip of the stylus was used. The tip is a plastic shaft that sits inside a casing of conductive rubber. It is a perfect fit for the rubber, holding it in shape, unlike the nuts and bolts used previously. Custom made washers held the pen tips in place. To improve the stability of the protractor an extra touch point was added (Figure 3 ). This design helped to provide a more stable base for the screen to tangible contact.

To improve the tangible to body contact a metal handle was added as an affordance to users as to where to hold the tangible. The handle also provided ease of movement. The wiring was kept the same, using copper wires to connect the touch points together via the conductive rubber tips.

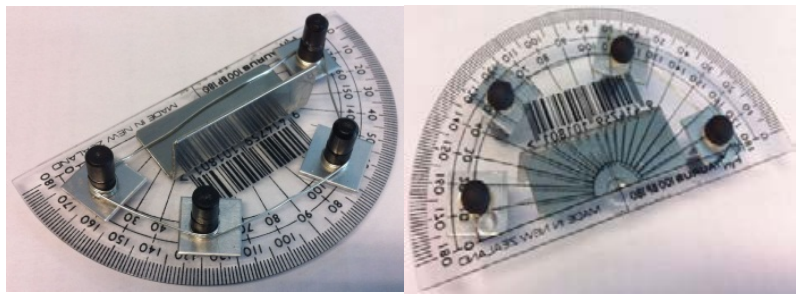
a. Top
b. Bottom

Figure 3: Final iteration tangible design

\section{TANGIBLE RECOGNITION}

Recognition of the tangible allows for further intelligence to be added to the tangible interaction. Thus far, we have concentrated on accurate identification of each tangible.

To identify a specific tangible object on a touch surface, its touch points are arranged to form a unique pattern - this serves as the tangible's unique ID. The patterns are based on the distance between each pair of points. When constructing a pattern the distances must be distinct; for example, a pattern that forms an isosceles triangle is not allowed because it has two or more identical distances between points. Identical distances make it impossible to determine the correct orientation of the tangible, with the obvious exception of regular shapes (e.g. circle, square, etc.).

There are two main phases for the recognizer: learning and recognition. The learning phase allows users to register and calibrate the tangibles so that they are identified by the system. Several details about each tangible are stored: the location of the touch points, the distances between touch points (i.e. its unique ID), the outline points of the tangible and the type of tangible (ruler, protractor or set square) as specified by the user. This data is used later to identify the tangible and draw its outline on the screen when it has been recognized.

Tangibles only need to be registered with the system once. To register the tangible (Figure 4a), the user places it on the registration screen and moves the black guidelines to delineate a bounding box. The user then specifies the type of tangible (ruler, set square, protractor) from a list.

During the recognition phase, touch input from the tangible currently in use is passed to the recognizer. The recognizer verifies its ID by calculating the distances between each touch point detected and comparing these to the previously saved distances for each calibrated tangible. The recognition adds (or subtracts) a degree of error tolerance (e) when comparing the distances between the detected touch points and the saved set of distances as follows:

$\mathrm{d}$ : detected distances; s: saved distances; $\mathrm{i}, \mathrm{j}$ : number of distances

For all $d_{i}$ and $s_{j}$, if:

$$
d_{i}-e<=s_{j}<=d_{i}+e ; \quad \text { then: } d_{i}=s_{j}
$$

If a match is found between all $d_{i}$ and $s_{j}$ then the tangible ID has been identified as $\mathrm{s}$. Our informal experiments showed that a $2 \mathrm{~mm}$ tolerance level is sufficient for maintaining a good rate of recognition.

The advantage of including a level of tolerance for the patterns is that $100 \%$ accuracy can be achieved by the recognizer, resulting in better usability. There is a tradeoff here, as calculating the position and orientation of the tangible is less accurate. To minimize its effect the user is provided with a visualization of the tangible on the screen (blue outline in Figure 4b). The visualization is rendered using the calculated position and orientation of the object; therefore it clearly shows any loss of precision. This gives the user important feedback on the recognition of the drawing instrument at that location and orientation. Using this information the user can adjust their drawings to follow the virtual outline.

The virtual outline of the tangible is rendered once the recognizer has confirmed the identity of the tangible. To do this a transformation matrix is formulated by finding the translation and rotation of 


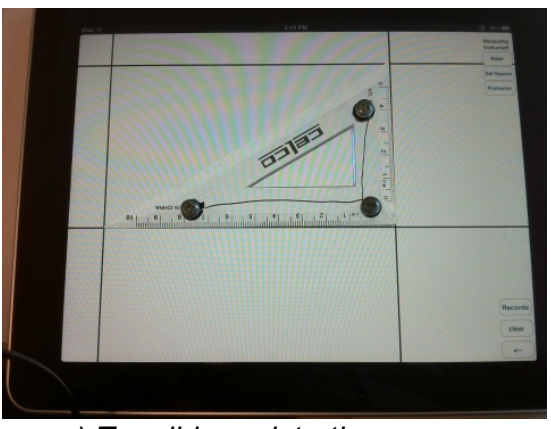

a) Tangible registration screen.

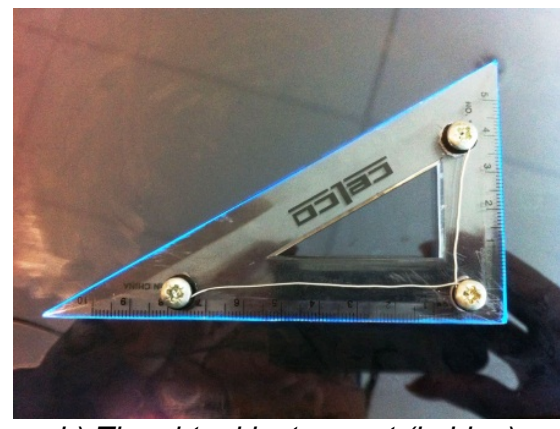

b) The virtual instrument (in blue) Figure 4: Tangible drawing application

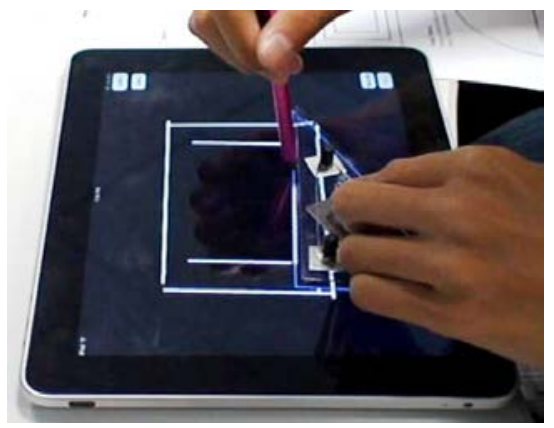

c) Drawing using a tangible object. the current touch points on the screen and comparing them to the previously saved touch point information for the object. The matrix is applied to the saved outline points so that a correctly orientated outline of the tangible is drawn on the screen - showing the virtual instrument directly under the recognized physical object (Figure 4b). This process is repeated each time the user moves the tangible.

We found that while moving a tangible on the screen, a touch point could be lost (lifted, or moved outside of the screen boundary), hindering usability. To improve this, recognition continues with only two points after the initial recognition of a three (or more) point tangible. A minimum of two points is needed to calculate the orientation of the tangible. This feature allows a point(s) to move off the screen without losing track of the tangible.

The recognition algorithm is cross platform (c++). We have successfully used it on both the Apple iPad and a Dell Windows 7 Tablet. Components related to the GUI, e.g. tangible registration, are platform dependent. We have two parallel implementations for iOS and Windows.

\section{DRAWING APPLICATION}

The tangible hardware and recognition algorithm were combined together in a drawing application. Our application allows the tangible ruler, protractor and set square to be used to construct drawings on a capacitive multi-touch screen. Given that this work is highly exploratory we have designed the application to have minimal functionality.

To construct drawings using a tangible drawing tool the user can slide their finger or stylus along the edge of the object that is placed on the screen. As the user draws, the strokes are detected and beautified lines (or curves) are rendered (Figure 4c). Any points that are not recognized as part of a tangible object are treated as drawing strokes.

\section{EVALUATION}

The goal of our evaluation was to assess whether this system has further potential by evaluating its usability. In particular, we wanted to determine whether people could use the tangible drawing tools to construct simple drawings with our application.

There were a total of ten participants in the study, six male and four female, aged between 18 and 35 . They were undergraduate and graduate students from a variety of backgrounds, including computer science, commerce and mathematics. Three participants used tablet devices frequently and three had used drawing applications on tablet devices occasionally. We asked participants to complete specific drawing tasks using the tangibles. The tangibles were registered by the researcher before the study. Each session lasted approximately 20 minutes.

Before the participants began they were introduced to the system and given time to familiarize themselves with the tools and do some practice tasks before the formal tasks began. Once the participants were comfortable with the system they were given a task sheet showing the diagrams they had to reproduce and the recommended tangible tool(s) to use. They were asked to draw eight lines intersecting through a single point and a house with a curved roof, a door and a window Figure 5. The first task was chosen to test how precisely users could draw strokes in relation to one another. The second task encouraged the use of all three tangibles. Participants proceeded to the next task when they were satisfied with their drawing. After the tasks were completed they filled out a questionnaire (using 5-point Likert scales); each question is shown in Table 1.

\section{Results}

A selection of sketches drawn by participants is shown in Figure 5. On observation of these drawings we can see that participants were able to use the tangible drawing tools to construct the required sketches. In addition, the results in Table 1 show that participants found it easy to learn how to use the drawing tools (mean rating: 4.7) and enjoyed completing the tasks (mean rating: 4). All participants agreed that the rate of recognition was good (mean rating: 3.9), except for one who was neutral. Six participants found it easy to use the 
Table 1: Questionnaire Results.

\begin{tabular}{|c|l|l|l|l|l|l|}
\hline & $\begin{array}{c}\text { Tasks were } \\
\text { enjoyable }\end{array}$ & $\begin{array}{c}\text { It is easy to use } \\
\text { drawing tools } \\
\text { on the tablet }\end{array}$ & $\begin{array}{c}\text { It is easy to } \\
\text { learn how to } \\
\text { use drawing } \\
\text { tools }\end{array}$ & $\begin{array}{c}\text { I think my } \\
\text { drawings were } \\
\text { accurate }\end{array}$ & $\begin{array}{c}\text { Rate of } \\
\text { recognition } \\
\text { was good }\end{array}$ & $\begin{array}{c}\text { I would like to use } \\
\text { tangibles to aid } \\
\text { drawing in the } \\
\text { future }\end{array}$ \\
\hline P1 & 4 & 4 & 4 & 4 & 4 & 3 \\
\hline P2 & 5 & 4 & 5 & 4 & 4 & 5 \\
\hline P3 & 4 & 4 & 4 & 2 & 4 & 4 \\
\hline P4 & 3 & 3 & 5 & 3 & 4 & 4 \\
\hline P5 & 4 & 4 & 5 & 4 & 4 & 3 \\
\hline P6 & 5 & 3 & 4 & 4 & 4 & 5 \\
\hline P7 & 3 & 4 & 5 & 4 & 3 & 3 \\
\hline P8 & 4 & 4 & 5 & 3 & 4 & 5 \\
\hline P9 & 4 & 3 & 3 & 3.4 & 3.9 \\
\hline P10 & 4 & 3 & Occasionally unwanted strokes were drawn when a \\
\hline Mean & 4 & 3.6 &
\end{tabular}
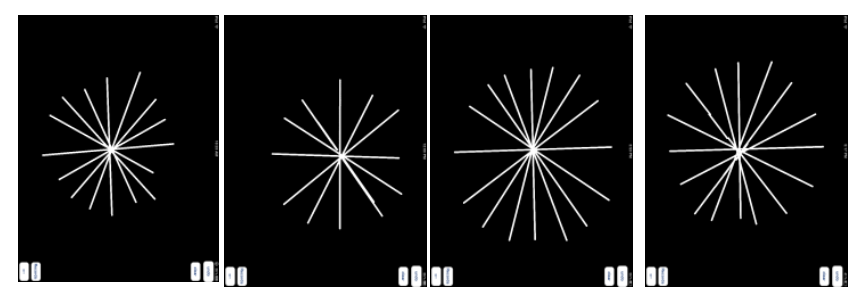

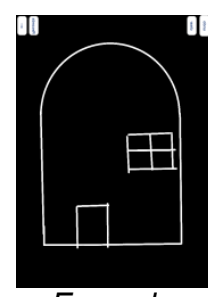

Example

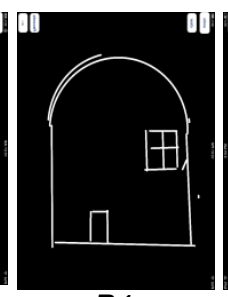

P4

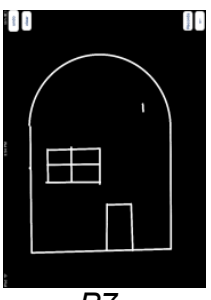

P7

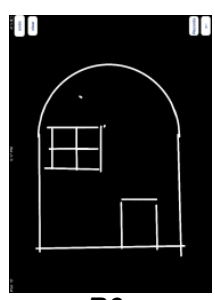

$P 9$
Figure 5: Task example and participant drawings

drawing tools on the tablet, the rest were neutral (mean rating: 3.6). Half of the participants said they would like to use tangibles to aid drawing on multitouch devices in the future; the remaining participants were neutral (mean rating: 3.8). These results indicate that our system has potential for further development.

Half of the participants agreed that their drawings were accurate, four were neutral and only one, participant four, did not believe that his drawing was accurate (mean rating: 3.4 ). We can see from the sketch in Figure 5, that participant four only drew six intersecting lines instead of eight for the first task and had a large unwanted stroke on the roof of the house. This participant spent the least amount of time (149 seconds) to complete the tasks; spending 44 s on task one and 105s on task two. This was well below the average times of $72.5 \mathrm{~s}$ and 170.7 for each task respectively. We also observed that he seemed to rush through the tasks. These factors may have contributed to the low accuracy rating given. On average, participants took a total of $243.2 \mathrm{~s}$ to complete the tasks.

We observed that continuous detection of the tangibles on the screen was problematic at times. tangible touch point was mistakenly treated as a drawing stroke - usually because all tangible touch points had not been detected. However, once detected, recognition of the tangibles was always correct.

\section{DISCUSSION}

It was easy for participants to learn how to use the tangible tools because they are familiar instruments. It does not require a deep learning curve for people to understand how to use them on a tablet device such as an iPad.

When a tangible has been detected by the system, recognition of the particular object, whether it is a ruler, protractor or set square, is $100 \%$ accurate. This indicates that our method of distinguishing tangibles using the unique ID patterns has been successful. This ability to accurately recognize a variety of tangibles suggests that it is feasible to use in applications which employ multiple tangible drawing tools such as school geometry and architectural drafting.

The main challenges lie in improving the design of the tangible hardware so that the touch point detection rates are higher. In some cases this may be a result of the user losing contact with the tangible. We also believe that the tangibles must have a more stable design where the gap between the screen and the object is minimized.

Another area where improvements can be made is in calculating the tangible location and orientation more precisely. Unfortunately the main limitation here is the hardware. The iPad, for example, reports the centre point position of a touch. Therefore the precise position of a touch point is difficult to determine as we don't know which part of the tangible's touch point was detected as the centre. This in turn affects the accuracy of tangible orientation computation. However, our visualization of the tangible on the display acts as an accurate 
guideline for the user, which can compensate for any discrepancies in the computation.

As this is the first study into the use of tangible drawing tools on capacitive multi-touch displays we purposefully built the application with a minimum amount of intelligence. Currently the application beautifies the lines drawn by the user and provides a virtual outline as a guide. This could be extended to include snapped corners where lines automatically meet to improve the aesthetics of the drawing. In addition, we could include visualizations relating to measurements that are made with the tangibles, such as the length or angle of lines as they are being drawn. We also plan to integrate geometry packages (Straesser 2002; Hohenwarter et al. 2007) into the system to add intelligent learning aspects to the tool. Our focus will be on functions that take advantage of the unique identification of each tool. With such intelligence, we believe this system has the potential to provide far more benefits than using traditional drawing tools on a screen or paper.

\section{CONCLUSION}

We have developed an innovative system that allows tangible drawing instruments to be used to construct drawings on capacitive multi-touch screens. The system is composed of three main components: the tangible hardware, the recognizer and the drawing application. Our evaluation shows that users are able to construct simple drawings easily with the tangible drawing tools, and the tangibles have a good rate of recognition with the touch screen. This project has produced a unique approach to creating geometric constructions. In the future this could be extended to interactive math education programs and architectural design with more intelligent drawing support.

\section{REFERENCES}

Chan, L., S. Müller, et al. (2012) CapStones and ZebraWidgets: sensing stacks of building blocks, dials and sliders on capacitive touch screens. Proceedings of the 2012 ACM annual conference on Human Factors in Computing Systems, Austin, Texas, USA, 5-10 May, pp. 2189-2192. ACM, New York.

Fails, J. A., A. Druin, et al. (2005) Child's play: a comparison of desktop and physical interactive environments. Proceedings of the 2005 conference on Interaction design and children, Boulder, Colorado, 8-10 June, pp. 48-55. ACM, New York.

Fitzmaurice, G. W., Ishii, H., Buxton, W. (1995) Bricks: Laying the Foundations for Graspable User Interfaces. Proceedings of CHI'95, Denver, Colorado, USA, 14-18 April, pp. 442-449. ACM Press/Addison-Wesley Publishing Co., New York.
Hohenwarter, M. and K. Jones (2007) Ways of linking geometry and algebra: the case of Geogebra. Proceedings of the British Society for Research into Learning Mathematics 27(3): pp. 126-131.

Inhelder, B. and J. Piaget (1964) The early growth of logic in the child. Harper \& Row, New York.

Marzola, E. S. (1987) Using Manipulatives in Math Instruction. Journal of Reading, Writing, and Learning Disabilities International 3: pp. 9-20.

Olson, I. C., Z. A. Leong, et al. (2010) It's just a toolbar!: using tangibles to help children manage conflict around a multi-touch tabletop. In Proceedings of the fifth international conference on Tangible, embedded, and embodied interaction (TEl '11), Funchal, Portugal, 22 - 26 January, pp. 29-36. ACM, New York.

Stanton, D., V. Bayon, et al. (2001) Classroom collaboration in the design of tangible interfaces for storytelling. In Proceedings of the SIGCHI conference on Human factors in computing systems (CHI '01), Seattle, Washington, 31 March 5 April, pp. 482-489. ACM, New York.

Straesser, R. (2002) Cabri-géomètre: Does Dynamic Geometry Software (DGS) Change Geometry and its Teaching and Learning? International Journal of Computers for Mathematical Learning 6: pp. 319-333.

Tuddenham, P., D. Kirk, et al. (2010) Graspables revisited: multi-touch vs. tangible input for tabletop displays in acquisition and manipulation tasks. In Proceedings of the 28th international conference on Human factors in computing systems ( $\mathrm{CHI}$ '10), Atlanta, GA, USA, 10-15 April, pp. 2223-2232. ACM, New York.

Ullmer, B. and H. Ishii (1997) The metaDESK: models and prototypes for tangible user interfaces. In Proceedings of the 10th annual ACM symposium on User interface software and technology (UIST '97), Banff, Canada, 14-17 October, pp. 223-232. ACM, New York.

Uttal, D. H., K. V. Scudder, et al. (1997) Manipulatives as symbols: A new perspective on the use of concrete objects to teach mathematics. Journal of Applied Developmental Psychology 18: pp. 37-54.

Wellner, P. (1991) The DigitalDesk calculator: tangible manipulation on a desk top display. Proceedings of the 4th annual ACM symposium on User interface software and technology, Hilton Head, South Carolina, United States, 11-13 November, pp. 27-33. ACM, New York.

Yu, N.-H., L.-W. Chan, et al. (2011) TUIC: enabling tangible interaction on capacitive multi-touch displays. In Proceedings of the conference on Human factors in computing systems ( $\mathrm{CHI}$ '11), Vancouver, BC, Canada, 7-12 May, pp. 2995-3004. ACM, New York. 